\title{
PRESUPUESTO PARTICIPATIVO EN LA CIUDAD DE LA PLATA: UN ESTUDIO COMPARADO A PARTIR DE LOS CAMBIOS POLÍTICOS
} STUDY BASED ON THE POLITICAL CHANGES 2014-2018

\author{
Ricardo Sebastián Piana \\ http://orcid.org/0000-0001-8743-8942 / r_piana@yahoo.es \\ Universidad Nacional de La Plata, \\ La Plata, Argentina.
}

DIEGO FURNARI

http://orcid.org/0000-0001-7513-5610 / furnaridiego@hotmail.com Universidad Católica de La Plata.

La Plata, Argentina.

\begin{abstract}
RESUMO
Pretende-se estudar comparativamente as políticas participativas na cidade de La Plata, Argentina, durante o período 2014-2018. O período escolhido inclui os dois últimos anos da administração do ex-prefeito Pablo Bruera, durante os quais as políticas de orçamento participativo foram intensificadas e os primeiros anos do novo líder comunal Julio Garro, quem decidiu modificar alguns aspectos substanciais. Este tipo de política, entendida como gestão municipal "aberta" à comunidade, tem sido apoiada por esforços de dois partidos políticos diferentes. Para fazer esta análise de estudo de caso, são estudados não apenas as ações, mas também os aspectos discursivos dos diferentes governos. Para além de certa continuidade, as mudanças parecem ser amplas: percebemos, como conclusão, como as mudanças processuais nas políticas participativas, mesmo fazendo uma certa continuidade discursiva, envolvem mudanças fundamentais na maneira de entender a relação entre governar e governar, compreender a realidade e agir de acordo com essa compreensão.
\end{abstract}

Palavras-chave: Governo aberto; Participação; Orçamento participativo; Transparência.

\begin{abstract}
It is intended to study comparatively participatory policies in the city of La Plata, Argentina, during the period 20142018. The chosen period includes the last two years of the former Mayor Pablo Bruera's administration, during which the participatory budgeting policies were intensified and the first years of the new communal leader Julio Garro, who decided to modify some substantial aspects. This type of policy, understood as city open gov to the community have been supported by two different political parties. To do this case study analysis, it is being studied not only the actions but also the discursive aspects of the different governments. Beyond the continuities, the changes seem to be wide: we notice, as conclusion, how the procedural changes in participatory policies, even do a certain discursive continuity, involve fundamental changes in the way of understanding the relationship between the governing and the governed, of understanding reality and of acting in accordance with that understanding.
\end{abstract}

Keywords: Open Government; Participation; Participatory Budget; Transparency. 


\section{RESUMEN}

Se pretende estudiar comparativamente las políticas participativas en la ciudad de La Plata, Argentina, durante el período 2014-2018. El período elegido comprende los dos últimos años de la gestión del ex Intendente Pablo Bruera durante la cual se intensificaron las políticas de Presupuesto Participativo y los primeros años del nuevo jefe comunal Julio Garro quien decidió modificar algunos aspectos sustanciales. Este tipo de políticas, entendidas como gestión municipal "abierta" a la comunidad han sido sostenidas por gestiones de distinto color político. Para el análisis del caso, se estudian no sólo las acciones sino también los aspectos discursivos de los distintos gobiernos. Más allá de las continuidades, los cambios parecen ser grandes: advertiremos, como conclusión, cómo los cambios procedimentales en las políticas participativas, aún una cierta continuidad discursiva, suponen modificaciones de fondo en la manera de entender la relación gobernante-gobernados, de entender la realidad y de actuar de conformidad a ese entendimiento.

Palabras clave: Gobierno Abierto; Participación; Presupuesto Participativo; Transparencia.

\section{SUMÁRIO}

INTRODUCCIÓN. 1 LOS ANTECEDENTES INSTITUCIONALES; 2 EL GOBIERNO ABIERTO COMO PARAGUAS PROTECTOR Y LEGITIMADOR; 3 PRESUPUESTO PARTICIPATIVO EN LA CIUDAD DE LA PLATA; 3.1 LOS aspectos discursivos; 3.2 Los aspectos Regulatorios; 3.3 Los Resultados; 3.4 Algunas Consideraciones Parciales; 4 CONSIDERACIONES FINALES; REFERÊNCIAS.

\section{INTRODUÇÃO}

El presente trabajo pretende describir comparativamente el presupuesto participativo en la ciudad de La Plata durante el período 2014-2018.

Dicho conjunto de políticas, enmarcadas hoy en las prácticas del gobierno abierto, se presentaron como uno de los ejes de campaña para el actual gobierno (en los niveles nacional, provincial y municipal). Se ha pretendido impulsar novedosas maneras de intervenir en el espacio público conjuntamente con la ciudadanía entendida como sujeto de derechos pero también de obligaciones. Desde esa doble perspectiva se insiste en generar las condiciones (instrumentos de política pública, piezas comunicacionales, discursos de campaña, entre otras) para que la ciudadanía se acerque y aproveche los beneficios de participar y colaborar en los procesos políticos que el gobierno de turno ofrece a la vez que se promueve, desde lo discursivo, una serie de reformas a políticas y prácticas prexistentes.

Esta tendencia no es nueva, pero ha encontrado en los últimos años un fuerte impulso desde diversas áreas, tanto por parte de los académicos como por los gobiernos, que encuentran en estas prácticas un mecanismo de legitimación para las políticas públicas.

Proponemos centrar el foco para estudiar estas políticas participativas en los gobiernos locales por varias razones. En primer lugar, porque en Argentina las experiencias participativas se han desarrollado inicialmente (y han encontrado interesantes espacios de desarrollo y estudio académico) en los gobiernos municipales. Los casos de Rosario, Morón, Ciudad de Buenos Aires y 
La Plata han sido municipios señeros en esta temática, pudiendo señalar como antecedes de estudio las investigaciones de Annunziata, 2011 y 2013; Balardini y Torrillate, 2009; Bloj, 2008; Carmona, 2011; Carmona y Martínez, 2014; Furnari, 2014; Lacarrieu, 2004; Pagani, 2015 y 2016; Plot, 2005; Pinillos y Signorelli, 2014; Quintar, 2009; Ramella y Galván, 2012; Romero, 2004; Santana, 2009; Schillagi, 2005; Sortino, 2013, entre otras.

Además, en el contexto de la actual crisis de representación política que influye sobre la baja percepción de los ciudadanos latinoamericanos sobre los resultados de sus democracias (Piana, 2014), se sostiene que en la detección de los problemas de política pública, cuanto más cerca del territorio esté puesto el análisis, más eficientes deberían ser los diagnósticos y, por tanto, mejores las soluciones propuestas. Tal como lo sostiene Uranga (2005:3):

Hoy las condiciones son otras, el contexto global es distinto y los modos de participación son también diferentes. Los partidos políticos tradicionales han dejado de ser por sí solos actores eficaces para la construcción democrática. Vivimos una profunda crisis de la partidocracia tradicional y aún hoy los liderazgos emergentes, surgidos de las nuevas prácticas, no logran consolidarse para constituirse en alternativas de poder. Es necesario pasar de una democracia de electores, a una democracia de participación, es decir, a una democracia de ciudadanos con capacidad de incidencia y poder real, partiendo del reconocimiento y el respeto por la diferencia.

La necesidad de fortalecer las democracias, encuentra hoy en el gobierno local la clave de bóveda para sostenerlo: la profundización de los procesos de descentralización de la política pública (de nación a provincia y de ésta última a los municipios) prioriza al régimen municipal porque brinda las mayores posibilidades de trabajar al lado del vecino-ciudadano desde un plano de proximidad y permite conocerlo para crear o afirmar vínculos de interacción para el trabajo comunitario y generar soluciones para los problemas cotidianos .

Ahora bien, qué distancia existe entre las consignas y las prácticas; esto es, cómo se trasladan estas prácticas discursivas a las acciones. Para intentar responder este interrogante sin pretender que del presente estudio se puedan inferir resultados generales- hemos tomando la política de presupuesto participativo de la ciudad de La Plata entre los años 2014 y 2018 . El municipio de La Plata, además de ser uno de los primeros gobiernos locales que inició este tipo de políticas, resulta relevante porque a pesar de los cambios políticos, se ha mantenido en las dos últimas gestiones. En efecto, a pesar de las fuertes críticas durante la campaña electoral a la gestión anterior, la actual conducción liderada por Julio Garro continuó la política del ex Intendente Pablo Bruera. 
Para el estudio de las continuidades y cambios se propondrá un camino descriptivo/comparativo en la aplicación, el diagnóstico, la visión, el rol ideológico y los objetivos de gestión. En la primera parte describiremos los antecedentes institucionales sobre las acciones en materia de reforma estatal en los últimos años. Luego se estudia el concepto de Gobierno Abierto como paraguas protector y legitimador de las actuales políticas de reforma. En la tercera sección se estudia el Presupuesto participativo en la ciudad de La Plata: los aspectos discursivos y regulatorios para luego presentar los resultados y algunas consideraciones parciales. Al finalizar el artículo se intentará comprender las motivaciones de la actual gestión y la existencia o no de un correlato entre el aspecto discursivo, ideológico y práctico a la luz de las nuevas estrategias de Gobierno Abierto.

\section{LOS ANTECEDENTES INSTITUCIONALES}

En la esfera política, como en ninguna otra, se ha tornado indispensable la definición de un marco conceptual que no sólo brinde certezas terminológicas respecto del camino a seguir, sino, y más importante, que brinde legitimidad a la multiplicidad de acciones/decisiones que se adoptan durante una gestión de gobierno. Ello se torna fundamental dado los altísimos niveles de desconfianza que la ciudadanía, en general, aún conserva respecto de las motivaciones de nuestros funcionarios, sobre todo, cuando se proponen cambios de fondo en las políticas públicas.

La llegada de una nueva gestión política de manera simultánea al nivel nacional, provincial y local brindó el contexto adecuado para que el gobierno defina una estrategia para alcanzar determinados objetivos de gestión modificando algunas prácticas: luego de asumir la presidencia, Mauricio Macri encabezó una serie de anuncios entre los cuales se destacaron la firma del Decreto $N^{\circ} 117 / 16$ mediante el cual se instruyó a los Ministerios, Secretarías y organismos desconcentrados y descentralizados dependientes del Poder Ejecutivo Nacional, a elaborar y presentar en un plazo no mayor a ciento ochenta (180) días ante el Ministerio de Modernización (órgano del cual depende el plan en cuestión) un “Plan de Apertura de Datos", cuyo principal objetivo está destinado a promover la difusión pública de información de los funcionarios a través de las declaraciones juradas así como datos respecto del uso de los fondos públicos y los resultados de gestión de los distintos organismos. La tendencia se profundizó con la aprobación del Decreto $N^{\circ}$ 434/16 (marzo de ese año) mediante el cual se aprobó el "Plan de Modernización del Estado” cuyos ejes son: 1) Plan de Tecnología y Gobierno Digital; 2) Gestión 
Integral de los Recursos Humanos; 3) Gestión por Resultados y Compromisos Públicos; 4) Gobierno Abierto e Innovación Pública; 5) Estrategia País Digital. Propiciada por el Poder Ejecutivo, en septiembre de ese mismo año se sancionó la Ley, registrado bajo el $n^{\circ} 27.275$, que regula el derecho de acceso a la información pública aún cuando las modificaciones que se introdujeron por decreto de necesidad y urgencia han sido cuestionadas. En julio de 2017, el gobierno nacional presentó ante la Alianza para el Gobierno Abierto, el 3er Plan de Acción Nacional de Gobierno Abierto, con compromisos de los 3 poderes del Estado Nacional, de los Organismos de Control Nacional y de 11 Provincias. La última iniciativa que puede tomarse como antecedente, es el lanzamiento de la "Plataforma Digital de Participación Ciudadana", la cual fue anunciada por la Secretaria de Modernización del Estado, Ciencia y Tecnología, Lucrecia Escandón, en el Encuentro Regional para las Américas de la Alianza para el Gobierno Abierto el cual promueve el intercambio de buenas prácticas entre los 70 países miembros y que se inauguró en Buenos Aires con la presencia del presidente Mauricio Macri en diciembre de 2017.

Estas líneas se replicaron en la mayoría de las provincias argentinas y los municipios gobernados por el partido Cambiemos de manera de demostrar un hilo conductor que, en materia de reforma administrativa, pretende impulsar la actual gestión.

En el caso de la provincia de Buenos Aires, la gobernadora María Eugenia Vidal, en febrero de 2016, aprobó el régimen de las declaraciones juradas patrimoniales de los funcionarios y agentes del Poder Ejecutivo, lo cual se profundizó, por Decreto $N^{\circ} 407 / 17$, al personal policial con jerarquía superior a subcomisario.

También en Provincia de Buenos Aires, se suscribió, en marzo del 2016, un acuerdo de cooperación con el Ministerio de Modernización de la Nación para la implementación del expediente digital (hoy Sistema de Gestión Documental Electrónica - GDEBA) con el objeto de agilizar los procesos administrativos con una inversión de más de 150.000 millones de pesos.

En julio de 2016, también impulsado por el Poder Ejecutivo provincial, la Legislatura aprobó el Plan Estratégico de Modernización de la Administración Pública de la Provincia de Buenos Aires, Ley $N^{\circ} 14.828$, destacándose en los fundamentos, que el "proyecto responde a la imperiosa necesidad de adecuar los procesos administrativos a los sistemas de calidad, herramientas informáticas y buenas prácticas de gestión administrativa actualmente vigentes a nivel regional y mundial". Ese mismo mes, a través del Decreto $\mathrm{N}^{\circ} 805 / 16$, se anunció la creación de un portal de datos abiertos para el Estado bonaerense a los efectos de sintetizar la información de los distintos organismos de gobierno. 
En abril de 2017 se anunció la creación de la Oficina de Fortalecimiento Institucional (OFI) cuyo principal objetivo radica en la concreción de los objetivos de transparencia, eficacia y transformación de la Administración Pública, en sintonía con las acciones del gobierno nacional.

La ciudad de La Plata, capital de la provincia de Buenos Aires, también hubo cambio de partido político en diciembre de 2015, en sintonía con el gobierno nacional y provincial, y de la mano de la nueva conducción se convirtió rápidamente en uno de los bastiones de la gestión provincial. Con una muy buena relación entre ambos ejecutivos, La Plata se transformó en un reducto clave para la consolidación del espacio Cambiemos y las decisiones tomadas desde el nuevo gobierno municipal así lo manifiestan.

En lo que nos atañe, el entonces candidato Julio Garro, como parte de su campaña manifestó su postura respecto a las nuevas tendencias en materia de reforma administrativa cuando anunciaba que su hipotética gestión se basaría en un gobierno abierto, transparente y que piense en la gente. Ya en la gestión, el responsable del ejecutivo municipal presentó en julio de 2017 el Portal de Datos Abiertos, un sitio que reúne información del sector público a disposición de la ciudadanía, el cual cuenta con el apoyo tanto del gobierno provincial como nacional. Sobre el portal de datos, el secretario de Modernización de la comuna, Federico Ortiz, señaló que:

Esta iniciativa responde al pedido expreso de nuestro intendente Garro de abrir el gobierno a los vecinos, utilizando las nuevas herramientas que nos ofrece la tecnología de la información y la comunicación. Tomamos la decisión de poner la información al servicio del ciudadano, estableciendo un nuevo paradigma de comunicación entre el sistema político y el vecino. ${ }^{1}$

Más allá de estas intenciones, no caben dudas que en la ciudad de La Plata el Presupuesto Participativo es la estrella de las políticas participativas, no sólo por la proporción del presupuesto general que se destina (98 millones de pesos en 2017, aproximadamente) sino también por la cantidad de personas que vienen participando directa e indirectamente y por la continuidad en el tiempo que ha tenido, independientemente de los cambios de gestión (tuvo sus inicios en 2008 con la gestión de Pablo Bruera y se encuentra en ejecución con Julio Garro).

Todos los antecedentes se encolumnan para identificar al Gobierno Abierto como el marco de referencia y paraguas legitimador de las propuestas de reforma administrativa del actual gobierno nacional, provincial y local.

1ORTIZ, Federico. Garro: Damos otro paso importante para seguir abriendo la gestión a los vecinos. Impulso Baires, La Plata, 17 jul. 2017. Disponible en: <http://www.impulsobaires.com.ar/nota/254094>. Acceso en: 9 jun. 2018. 
Veremos en el próximo apartado, qué es lo que se entiende como Gobierno Abierto y por qué identificamos a las políticas de presupuesto participativo, aún con un origen anterior a este nuevo paradigma, como parte de estas políticas.

\section{EL GOBIERNO ABIERTO COMO PARAGUAS PROTECTOR Y LEGITIMADOR}

¿Qué es este nuevo concepto de Gobierno Abierto que hoy engloba a todas las políticas públicas de modernización y genera tanto adeptos? A partir de la iniciativa del entonces presidente de los Estados Unidos Barack Obama, este enfoque no se centra ya en los aspectos tecnológicos (aunque la tecnología sí importa), sino en la participación, colaboración y transparencia junto a otros conceptos claves como el de rendición de cuentas y la aperturadisponibilidad de la información pública. Como señala la Declaración de Gobierno Abierto suscripta en el marco de la Alianza de Gobierno Abierto, más conocida por su acrónimo en inglés, OGP, varios gobiernos de la región se han comprometido a aumentar la disponibilidad de información sobre las actividades gubernamentales; apoyar la participación ciudadana; aplicar los más altos estándares de integridad profesional en todos nuestros gobiernos y aumentar el acceso a las nuevas tecnologías para la apertura y la rendición de cuentas.

En nuestro ámbito, se ha señalado que el Gobierno Abierto es...

[...] el conjunto de mecanismos y estrategias que contribuye a la gobernanza pública y al buen gobierno, basado en los pilares de la transparencia, participación ciudadana, rendición de cuentas, colaboración e innovación, centrando e incluyendo a la ciudadanía en el proceso de toma de decisiones, así como en la formulación e implementación de políticas públicas, para fortalecer la democracia, la legitimidad de la acción pública y el bienestar colectivo. ${ }^{2}$

Sin embargo, es importante aclarar, como lo señala Álvaro V. Ramírez-Alujas, que

El término Gobierno abierto no es nuevo. A fines de los años 70 del siglo XX se usó por primera vez en el espacio político británico y en su concepción original, trataba diversas cuestiones relacionadas con el secreto de Gobierno e iniciativas para "abrir las ventanas" del sector público hacia el escrutinio ciudadano, con el objeto de reducir la opacidad burocrática. En la actualidad, se ha posicionado como un nuevo eje articulador de los esfuerzos por mejorar las capacidades del

${ }^{2}$ CONSEJO LATINOAMERICANO DE ADMINISTRACIÓN PARA EL DESARROLLO - CLAD. Carta Iberoamericana de Gobierno Abierto. 2016. Disponible en < https://www.clad.org/images/declaraciones/CIGA-Octubre2016.pdf>. Acceso en: 10 sep. 2018. 

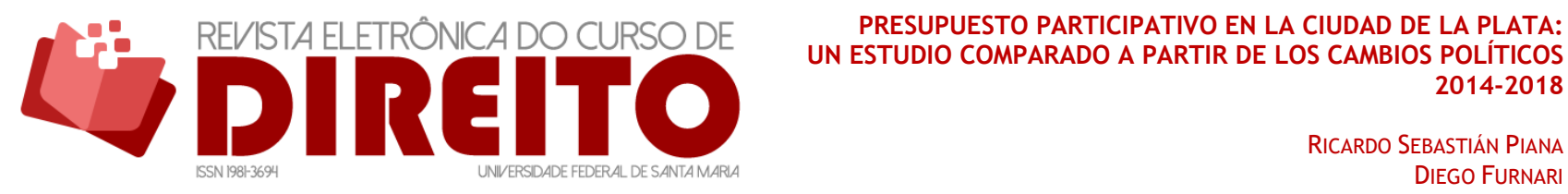

Gobierno y modernizar las administraciones públicas bajo los principios de la transparencia y apertura, la participación y la colaboración. ${ }^{3}$

En igual sentido, Oscar Oszlak, afirma que "El gobierno abierto no es un nuevo desarrollo tecnológico: es una verdadera filosofía acerca de cómo gobernar y de cuál es el rol que juegan el gobierno y los ciudadanos en la gestión pública y en sus resultados" (Oszlak, 2012:3). En efecto, pese a la intención de presentar estas iniciativas como una revolución administrativa, la realidad es que se viene hablando de estos temas desde hace más de 40 años y muchos de los países económicamente más desarrollados ya han impulsado este tipo de experiencias más allá del nombre que se les hayan asignado.

Resulta evidente que los tres ejes del Gobierno Abierto, transparencia, colaboración y participación, son los centrales pero no los únicos. La revolución tecnológica (globalización de por medio) y el proceso de mediatización de la política por el cual los medios impusieron su lógica en la construcción de la realidad, obliga a considerar la incorporación de nuevas herramientas tecnológicas que, por un lado, acortan la distancia entre gobernante y gobernado, y por otro lado, brindan un mayor y mejor acceso a la información pública, así como nuevos canales de interacción entre los vecinos. Justamente, es imposible desconocer que el Gobierno Abierto es precedido por otra "moda" en materia de reforma administrativa como la de Gobierno Electrónico (Piana y Furnari, 2015). Sabemos, sin embargo, que la sola existencia de las nuevas tecnologías (plataformas digitales, web 3.0, redes sociales, entre otros) no resultan suficientes si los ciudadanos (usuarios) no están preparados para el aprovechamiento integral de las mismas; tampoco cuando estas tecnologías sólo reproducen los procesos en papel sin aportar aspectos verdaderamente innovadores.

Para que el Gobierno Abierto sea una verdadera práctica es también esencial la apertura de canales de dialogo entre el gobierno y los ciudadanos: los canales tradicionales (comunicación telefónica, reclamos presenciales, etc.) ya no son suficientes. Es indudable que los gobiernos han expandido las alternativas para que el ciudadano peticione ante las autoridades, para que realice sus reclamos, genere sus espacios de diálogo y para que proponga sus ideas. Ahora bien, y en sintonía con la reserva realizada con anterioridad, la mera predisposición o puesta en marcha de canales institucionales para el diálogo no convierte mágicamente a un sistema administrativo en un gobierno abierto a su ciudadanía. La comunicación implica un compromiso consiente entre los participantes para poder avanzar en

\footnotetext{
${ }^{3}$ RAMÍREZ-ALUJAS, Álvaro. Gobierno abierto y modernización de la gestión pública: tendencias actuales y el (inevitable) camino que viene. Reflexiones seminales. Revista Enfoques, Vol. IX, №15, 2011, p. 101.
} 
una relación fructífera y de doble vía. Es decir, que además de la existencia de canales oficiales y de una predisposición discursiva del gobierno, los ciudadanos deben querer esa vinculación y también considerarla y valerse de ella por ser oportuna y útil para sus objetivos.

Queda expuesto que los tres componentes no pueden ser considerados sin analizar un elemento fundamental y que los amalgama: el rol del ciudadano. En tal sentido

[...] no es concebible la participación de la sociedad civil en el diseño, puesta en marcha y evaluación de las políticas estatales, a menos que ésta haya sido empoderada. El empoderamiento implica que el ciudadano conoce sus derechos individuales y los colectivos, la forma en que se puede obtener la garantía de su ejercicio y la capacidad de análisis de la información pertinente, así como su capacidad de agencia, o sea, de ser o hacer aquello que se tiene razones para valorar; y que aún empoderado, el ciudadano valora la participación política y tiene la voluntad de ejercerla. ${ }^{4}$

El ciudadano debe considerarse a sí mismo como un actor activo en la relación con el gobierno y propender a la co-producción de las decisiones públicas. Es decir, que no sólo depende de la voluntad del Estado sino que también de la voluntad, predisposición o decisión del ciudadano de participar de las propuestas que el gobierno o sus conciudadanos, se le ponen a su consideración. Si los depositarios de esta filosofía no consideran importante u oportuno hacer uso de la misma, no tendría sentido verdadero ninguna iniciativa ya que no se trata de crear estructuras unidireccionales o vacías de contenido sino de generar las condiciones necesarias para una mejor democracia y una administración más eficiente, transparente y participativa.

En nuestra Región la convivencia (poco sana) entre un modelo burocrático weberiano incompleto y la necesidad de avanzar hacia esquemas de tipo gerencial (al mejor etilo de las reformas aplicadas en Nueva Zelanda), nos enfrenta con un modelo amorfo, extraño y sumamente compleja dentro de la cual las actuales gestiones gubernamentales pretenden incluir nuevos enfoques y propuestas desde la óptica del Gobierno Abierto. Como también ha señalado Oszlak (2014) en otro trabajo que analiza las distintas iniciativas enmarcadas en las políticas de Gobierno Abierto, podría inferirse que los países no latinoamericanos, han avanzado más en la implementación de iniciativas que los de Latinoamérica aún cuando éstos presentan muchas propuestas. Por eso, y frente al carácter necesariamente discursivo de estas políticas,

\footnotetext{
${ }^{4}$ OSZLAK, Oscar. Gobierno Abierto: Promesas, supuestos, desafíos. VIII Conferencia anual Inpae 2012: gobierno abierto: por una gestión pública más transparente, participativa y colaborativa. San Juan de Puerto Rico, Puerto Rico, 2012. Anales electrónicos, p. 16.
} 
entendemos que en nuestro contexto es más relevante analizar prácticas y no sólo planes o programas pues una parte importante de éstos no pasan de las buenas intenciones.

Las políticas de presupuesto participativo nos ofrecen una perfecta oportunidad para estudiar las acciones concretas de Gobierno Abierto en la esfera local: hoy identificadas como políticas de Gobierno Abierto, aún cuando hayan iniciado antes que este vocablo tomara fuerza teórica, el presupuesto participativo aporta la suficiente prueba empírica más allá de los aspectos discursivos. Este análisis cobra mayor relevancia si tomamos en cuenta el cambio de gobierno acaecido en 2015 en los tres niveles de gobierno.

Para realizar nuestro estudio, se analizarán las prácticas locales en la ciudad de La Plata desde el punto de vista discursivo primero, y práctico luego; esto es, lo que las dos gestiones de gobierno en análisis (Bruera y Garro) fueron prometiendo y realizando durante el período en cuestión para poder arribar a una conclusión sobre el resultado de las políticas basada en la comparación entre modelos de gestión y gobierno que, en principio, se han planteado como diferentes.

\section{PRESUPUESTO PARTICIPATIVO EN LA CIUDAD DE LA PLATA}

Como se intentó dejar en claro en los párrafos anteriores, las nuevas estrategias de Gobierno Abierto se apoyan fuertemente (aunque no de manera exclusiva ni excluyente) en las herramientas, canales o modalidades que permiten la participación de los ciudadanos (individual o colectivamente a través de las distintas formas que toma la sociedad civil) en las diferentes instancias de la política pública de los gobiernos.

Resulta difícil reconocer instancias concretas de políticas públicas participativas en la Argentina anteriores a la llegada del Presupuesto Participativo el cual fue, por mucho tiempo, sinónimo casi excluyente de participación en la gestión pública.

La ciudad de La Plata fue una de las pioneras junto a Rosario y la Ciudad Autónoma de Buenos Aires al instalar el Presupuesto Participativo en el año 2008 y que ha tenido continuidad hasta la actualidad. ¿Pero qué se ha dicho y cómo se justifica/legitima el presupuesto participativo?

Según María Cristina Mata:

El conjunto de lo decible -que obviamente incluye lo no dicho- como podemos denominar al discurso social, es evidentemente un conjunto articulado a partir de disposiciones que revelan un orden establecido. Dentro del mismo las posibilidades de variación son tan amplias o estrechas según sean las condiciones 
que regulan su producción. Porque lo decible no se restringe a unos ciertos temas y modos expresivos, sino que incluye además un conjunto de disposiciones explícitas o implícitas -pero siempre legitimadas socialmente- acerca de los sujetos habilitados para proferir determinados discursos sociales, acerca de los lugares desde los que ellos pueden ser enunciados, acerca de los modos en que ellos pueden y deben circular y ser recibidos. ${ }^{5}$

Retomamos las preguntas, para intentar responder ¿cómo era el esquema de funcionamiento del Presupuesto Participativo (hasta 2015) y cómo es hoy? y ¿qué resultados cuantitativos ofrecen estas políticas en perspectiva comparada?

\title{
3.1. Los aspectos discursivos
}

Ya hemos señalado que las políticas de presupuesto participativo se dieron inicio en 2008 con la recién arribada gestión de Pablo Bruera. Como ha señalado María Laura Pagani:

\begin{abstract}
En ese momento, se instala fuertemente una modalidad de campaña a partir de caminatas que lideraba el propio Bruera y la línea de atención gratuita "Bruera te escucha", que recepcionaba reclamos que se intentaban resolver desde el Consejo Deliberante. Estas dos modalidades perduraron hasta la campaña del 2007, en la que Bruera logra ser electo con el $25,7 \%$ de los votos, con una diferencia de $7 \%$ con su rival Alak (que se presentaba a la cuarta reelección).

Ese bajo umbral electoral lo llevó a implementar una batería de políticas tendientes a cooptar el apoyo de un espectro electoral más amplio: en ese contexto nacen las políticas de presupuesto participativo junto con otras de visible impacto como la mejora de plazas, residuos secos y el "Servicio Municipal 72 horas" $^{6}$.
\end{abstract}

Según el entonces intendente, el presupuesto participativo era parte de un cambio político en torno a una forma de gestionar más abierta y expresó en el acto inaugural de su política de presupuesto participativo que: "Dijimos que veníamos a cambiar la forma de administrar el Municipio a partir de la idea de una comuna de puertas abiertas, sin exclusiones y este Presupuesto Participativo es un paso importante que estamos dando hacia ese objetivo" "En

\footnotetext{
5 MATA, Ma. Cristina. Nociones para pensar la comunicación y la cultura masiva. Buenos Aires, Argentina: Modulo 2, Curso de Especialización Educación para la comunicación-La Crujía, 1985, p. 9.

${ }^{6}$ PAGANI, Ma. Laura. Vos proponés, vos decidís: Presupuestos participativos y participaciones ciudadanas en La Plata y Morón 2006-2014. Tesis de doctorado en Ciencias Sociales. Facultad de Humanidades y Ciencias de la Educación, UNLP, 2015. p. 107.

${ }^{7}$ BRUERA, Pablo. Dijimos que veníamos a cambiar la forma de administrar el Municipio a partir de la idea de una comuna de puertas abiertas, sin exclusiones y este Presupuesto Participativo es un paso importante que estamos dando hacia ese objetivo. Diario El Día. La Plata, 22 febr. 2008. Disponible en: < https://www.eldia.com/nota/2008-2-22-con-criticas-bruera-lanzo-el-presupuesto-participativo >. Acceso en: 9 jun. 2018.
} 
igual sentido, el entonces Director ejecutivo del presupuesto participativo, Diego Santana (2009:3) expresó:

El Presupuesto Participativo se propone como un medio para alcanzar dos objetivos intrínsecamente relacionados: proponer nuevas formas de gestión pública que garanticen su eficiencia a través de la intervención de los ciudadanos y profundizar las bases democráticas del régimen, a través de una mayor participación en cuestiones estrechamente vinculadas con la vida cotidiana.

Otro de los objetivos de la implementación del Presupuesto Participativo era el de acercar la gestión municipal a los vecinos, el mismo fue cumplido de manera total, a partir de la generación de Asambleas Populares extraordinarias. Las mismas fueron fruto de los debates que surgían de los vecinos, quienes planteaban distintas dificultades en diversas áreas de gestión local. A partir de la propuesta de uno de los vecinos se generaba una fecha en la cual el funcionario responsable del área se presentaba para comunicar y debatir junto a los vecinos las políticas que su área de gestión estaba desarrollando en la zona, dichas Asambleas permiten reorganizar la gestión local, maximizando los recursos.

Pero más allá de estas declaraciones, hay muy pocas menciones a esta política durante los años de estudio tanto por parte del entonces Intendente Bruera como de sus funcionarios.

La gestión de Julio Garro, en sintonía con la decisión política del gobierno nacional y provincial, inició su gestión con el propósito de realizar una gestión más ágil, eficiente y trasparente. La adopción de los presupuestos fundamentales del Gobierno Abierto fue uno de los ejes de esta decisión y el presupuesto participativo nuevamente se transformó en la punta de lanza para que la gestión se mostrarse más abierta a la recepción y participación del vecino, potenciando ahora (y como elemento diferenciador), el uso de las TICs.

En tal sentido, en abril de 2017, el Intendente Julio Garro anunció el relanzamiento de dicha política pública (en 2016 no se ejecutó) y se manifestó señalando que: "Queremos que el vecino se involucre, participe y sea parte de un gobierno abierto"8. Esta nueva mirada implicó un cambio de forma, pero, como veremos más adelante, también de fondo en la manera de pensar y ejecutar el Presupuesto Participativo.

Aún cuando en su campaña electoral y los primeros meses de gestión se promovió la revalorización de las herramientas del gobierno abierto como una manera de transparentar la gestión municipal y de acercarse a la ciudadanía, resultó fuertemente contradictorio que durante el 2016 no se llevara adelante el presupuesto participativo, por primera vez desde sus

8 GARRO, Julio. Con críticas a la gestión anterior, Garro presentó la nueva etapa del Presupuesto Participativo. Realpolitik. La Plata, 18 abr. 2017. Disponible en: < https://realpolitik.com.ar/nota/24045/con_criticas_a_la_gestion_anterior_garro_presento_la_nueva_etap a_del_presupuesto_participativo/> Acceso en: 12 may. 2017. 
inicios, sin explicación pública alguna: no existió comunicación oficial por parte de las autoridades, dejando sin espacio a muchos vecinos que año a año venían participando e interesándose por esta posibilidad. Consultado el actual director del Presupuesto Participativo, Emiliano Calanna", explicó que "la no implementación del Presupuesto Participativo durante el 2016 respondió a la necesidad del Municipio de auditar los procedimientos que se venían implementando así como las obras votadas en el 2015".

En efecto, la nueva gestión consideraba que algunas obras no habían sido evaluadas desde el punto de vista técnico haciendo peligrar su factibilidad. A su vez, entendían que la modalidad mediante la cual se venía implementando esta política pública (asambleas barriales y votación presencial) excluía a muchos vecinos y perjudicaba la transparencia de la misma ya que el vecino votaba en cualquier delegación o barrio, independientemente de su domicilio legal lo cual, en palabras del entrevistado, "beneficiaba a los punteros políticos" que movilizaban a la gente de acuerdo a la obra que quisiesen promover.

En vistas del diagnóstico realizado por el gobierno municipal durante los primeros meses del 2016, se decidió realizar algunos cambios tanto de forma como de fondo para el Presupuesto Participativo 2017, entre las que destaca un portal web donde los vecinos votan propuestas realizadas por el municipio. Según el propio Intendente Julio Garro en declaraciones a los medios señaló respecto del Presupuesto Participativo 2017 que es una herramienta "más transparente e inclusiva" y que "promueve una participación directa mediante la cual los vecinos pueden transformar su comunidad y contribuir en la distribución de los recursos públicos del Municipio" 10 .

En palabras de Emiliano Calanna, "se decidió que los vecinos propongan y voten los proyectos directamente desde la plataforma digital (desde dispositivos móviles, tablets o pc) desde la comodidad de su hogar o espacio de trabajo, facilitando el acceso de todos"11.

Pero no todas las voces fueron contestes en cuanto a la apertura que proporciona este nuevo canal e instancia de participación. Carlos Sortino, funcionario a cargo la organización y el desarrollo del Presupuesto Participativo de La Plata entre los años 2008 y 2013, sostuvo que:

[...] el Presupuesto Participativo es una práctica que puede ser utilizada como una simple herramienta de marketing o como una estrategia de inclusión e innovación políticas. En La Plata, a partir de la gestión de Cambiemos, se optó

\footnotetext{
${ }^{9}$ CALANNA, Emilio. Entrevista concedida a FURNARI, Diego, 14 de febrero de 2017.

${ }^{10}$ GARRO, Julio. Los vecinos de La Plata ya pueden votar online las obras para su barrio. ADN Digital, La Plata, 20 abr. 2017. Disponível em: <http://andigital.com.ar/municipios/item/60371-los-vecinos-de-laplata-ya-pueden-votar-online-las-obras-para-su-barrio>. Acceso en: 9 jun. 2018.

${ }_{11}^{11}$ CALANNA, Emilio. Entrevista concedida a FURNARI, Diego, 14 de febrero de 2017.
} 


\section{UDIREITO}

PRESUPUESTO PARTICIPATIVO EN LA CIUDAD DE LA PLATA: UN ESTUDIO COMPARADO A PARTIR DE LOS CAMBIOS POLITICOS 2014-2018

RICARDO SEBASTIÁN PIANA DIEGO FURNARI

por lo primero: hacerlo exclusivamente vía internet, sin debate público, sin encuentro de personas en su propio territorio, es decir: un simulacro. $Y$ el empoderamiento del pueblo, de esta manera, no puede materializarse, simplemente porque lo único que se le ofrece es confianza en sus representantes y paciencia para lograr sus demandas. ${ }^{12}$

\subsection{Los aspectos regulatorios}

En el primer esquema de presupuesto participativo, reglamentado por Decreto $\mathrm{N}^{\circ}$ $343 / 08$, podían intervenir con voz todas las personas mayores de 16 años y con voto las mayores de 18 , siendo necesario un quorum de al menos 20 personas o agrupaciones para constituir la Asamblea Popular.

Se preveían 4 Asambleas: la primera de ellas, simultánea en 35 zonas con carácter informativo sobre el mecanismo de funcionamiento del Presupuesto Participativo, la organización del debate, la votación de los proyectos y la ejecución de las obras. En la segunda y tercer Asamblea se debatirían los proyectos. Entre una reunión y otra, el equipo técnico del Consejo de Presupuesto Participativo se ocupaba de definir el proyecto más adecuado, en forma conjunta con el área pertinente del Estado municipal según el tipo de propuesta. El equipo técnico del Consejo de Presupuesto Participativo se ocupaba de definir la viabilidad técnica, jurídica y económica del proyecto presentado y, en su caso, presentar a los vecinos reunidos en asamblea un proyecto alternativo. En la cuarta y última Asamblea se votarían los Proyectos definitivos que formarán parte de la semana de las Muestras Populares del Presupuesto Participativo

Como se ha señalado, la cuarta Asamblea Popular era la etapa del cierre de los proyectos: se votarían hasta 20 propuestas pudiendo participar en ésta aquellos vecinos e Instituciones que hayan participado de todas las Asambleas Populares de la zona. La segunda votación se llevaría a cabo en la etapa de las Muestras Populares respecto de las 20 propuestas, votación en la que podrían participar todos los vecinos de la zona mayores de 18 años incorporados al último padrón electoral. Finalmente, los recursos del Presupuesto Participativo se asignarían a las obras siguiendo ese orden, hasta que los recursos zonales alcancen definiendo la norma que este orden no podría ser modificado bajo ningún concepto.

Desde sus inicios ésta política pública casi no sufrió cambios estructurales hasta que en el año 2013,

[...]desde el Consejo del Presupuesto Participativo, se comenzó a implementar una nueva herramienta participativa cuyo objeto es establecer un marco

${ }^{12}$ SORTINO, Carlos Entrevista concedida a FURNARI, Diego, 01 de junio de 2018. 


\title{
UDIREITO
}

\author{
PRESUPUESTO PARTICIPATIVO EN LA CIUDAD DE LA PLATA: \\ UN ESTUDIO COMPARADO A PARTIR DE LOS CAMBIOS POLÍTICOS \\ 2014-2018 \\ RICARDO SEBASTIÁN PIANA \\ DIEGO FURNAR
}

regulatorio para las reuniones y procedimientos que se desarrollen en el marco del presupuesto y brindarle al vecino la posibilidad de participar en la elaboración o modificación del Reglamento Participado. Esta herramienta brinda posibilidades para que los vecinos puedan colaborar con el municipio para mejorar diariamente los instrumentos normativos que regulan al Presupuesto Participativo. De esta manera se profundiza la decisión política de otorgarle al vecino la mayor cantidad de espacios de participación y construcción social de sentido ${ }^{13}$.

Sin embargo, y aún cuando fuera un cambio positivo porque introducía una herramienta útil a los efectos de regular los procesos participativos e integrar en esa regulación a los propios vecinos, no hubo modificación alguna al Decreto del Ejecutivo.

Con el cambio de gobierno a fines de 2015, una nueva vuelta de tuerca se va a dar en materia de las pautas para decidir el presupuesto participativo que se plasmarían recién en 2017. En primer lugar, se hizo hincapié en la incorporación de nuevas tecnologías que hiciesen más accesible al vecino su participación y ampliase la transparencia de la política pública. Para ello se trabajó en una nueva plataforma digital (página web) que reemplazó a la anterior. La página actual ${ }^{14}$ se presenta más dinámica, con videos de la implementación de obras y tres simples pasos para el vecino: “¿Dónde vivís?” (para que el ciudadano confirme su lugar de residencia); “Elegí tu proyecto" (para poder proponer alternativas de política pública); y "Votá” (para que el ciudadano elija entre los diferentes proyectos propuestos por los vecinos de su barrio).

Otro cambio interesante en términos de apertura del gobierno fue la puesta en marcha de un portal de datos abiertos en donde el vecino puede consultar diferente tipos de información (datos demográficos, económicos, turísticos, etc.), y entre ellos, lo que respecta al Presupuesto Participativo ${ }^{15}$.

Estos cambios trajeron un cambio de suma importancia en comparación con el modelo anterior: la eliminación de las asambleas barriales como espacio donde se discutían las problemáticas barriales y se proponían las alternativas que luego evaluaba el municipio. Esos tres momentos de dialogo entre vecinos y autoridades municipales dejó de existir para ser reemplazado por la plataforma y la posible propuesta por parte del propio gobierno que, en

\footnotetext{
${ }^{13}$ FURNARI, Diego. Un análisis Comunicacional sobre el presupuesto participativo en el barrio Altos de Hernández: Construyendo procesos de integración. Tesis de la Maestría en Planificación y Gestión de Procesos Comunicacionales. Universidad Nacional de La Plata, Buenos Aires, Argentina, 2014, p. 84.

${ }^{14} \mathrm{LA}$ PLATA (ciudad). Presupuesto participativo. Disponible en: $<$ https: / / presupuestoparticipativo.laplata.gob.ar>. Acceso en: 6 mar. 2018.

${ }^{15}$ LA PLATA (ciudad). Datos abiertos. Disponible en: <https://www.datos.laplata.gov.ar>. Acceso en: 18 nov. 2018.
} 
PRESUPUESTO PARTICIPATIVO EN LA CIUDAD DE LA PLATA: UN ESTUDIO COMPARADO A PARTIR DE LOS CAMBIOS POLÍTICOS 2014-2018

Ricardo SEBASTIÁN PIANA DIEGO FURNARI

palabras del propio jefe Comunal, puede presentar propuestas aún cuando la primera plataforma no dejaba en claro cómo, cuándo ni dónde.

Un dato, por demás interesante en este cambio de prácticas, es que no se pudo detectar, desde la propia página del Digesto Municipal y de diversas consulta hechas a concejales de La Plata, norma alguna que haya reemplazado al Decreto sancionado durante el período de Bruera.

\subsection{Los resultados}

En el siguiente cuadro veremos los resultados del presupuesto participativo a partir de sus indicadores más relevantes:

Cuadro I - Indicadores presupuesto participativo - La Plata 2008 a 2018

\begin{tabular}{|c|c|c|c|c|c|c|c|c|c|c|c|}
\hline Indicadores & 2008 & 2009 & 2010 & 2011 & 2012 & 2013 & 2014 & 2015 & $2016^{16}$ & $2017^{17}$ & $2018^{18}$ \\
\hline $\begin{array}{l}\text { Presupuesto } \\
\text { asignado }^{19}\end{array}$ & 7 & 14 & 20 & 40 & 100 & 120 & 125 & 150 & - & 98 & $\cdots-\cdot-$ \\
\hline $\begin{array}{l}\text { Proporción s/ } \\
\text { Presupuesto } \\
\text { General }\end{array}$ & $2,1 \%$ & $3,4 \%$ & $3,7 \%$ & $6,1 \%$ & $8,8 \%$ & $9,2 \%$ & $\cdots-$ & $\cdots$ & $\cdots-\cdot$ & $\cdots-\cdot$ & $\cdots-\cdot$ \\
\hline $\begin{array}{l}\text { Proyectos } \\
\text { presentados }\end{array}$ & 513 & 341 & 368 & 255 & 275 & 142 & 131 & 150 & $-\cdots-\cdot$ & 204 & $\cdots$ \\
\hline $\begin{array}{l}\text { Proyectos } \\
\text { elegidos }\end{array}$ & 39 & 40 & 43 & 40 & 40 & 40 & 30 & 30 & $\cdots-\cdot-$ & 76 & -..-- \\
\hline $\begin{array}{l}\text { Proyectos } \\
\text { Infraestructur } \\
\text { a Urbana }\end{array}$ & $64,9 \%$ & $48,5 \%$ & $61,2 \%$ & $77,5 \%$ & $83,5 \%$ & $87,5 \%$ & $97 \%$ & $94 \%$ & $\cdots-\cdot$ & $94,2 \%$ & $\cdots-\cdot$ \\
\hline $\begin{array}{l}\text { Proyectos } \\
\text { Acción } \\
\text { Comunitaria }\end{array}$ & $35,1 \%$ & $51,5 \%$ & $38,8 \%$ & $22,5 \%$ & $16,5 \%$ & $12,5 \%$ & $3 \%$ & $6 \%$ & $\cdots-\cdot$ & $5,8 \%$ & $\cdots-\cdot-$ \\
\hline $\begin{array}{ll}\text { Asistencia } & \text { a } \\
\text { asambleas } & \end{array}$ & 3.342 & 3.200 & 5.230 & 2.682 & 3.570 & 2.150 & 2.528 & 6.000 & $\cdots$ & $\cdots-\cdot-$ & $\cdots-\cdot-$ \\
\hline $\begin{array}{l}\text { Asistencia a } \\
\text { Consulta } \\
\text { Popular }\end{array}$ & 17.686 & 29.574 & 45.037 & 49.561 & 51.104 & 52.017 & 32.198 & 34.000 & $\cdots$ & $\begin{array}{l}20.000 \\
20\end{array}$ & $\cdots-\cdot-$ \\
\hline $\begin{array}{l}\text { Participación } \\
\text { s/ población }{ }^{21}\end{array}$ & $3,8 \%$ & $6,3 \%$ & $9,6 \%$ & $9,9 \%$ & $10,2 \%$ & $10,4 \%$ & $6,5 \%$ & $8 \%$ & $\cdots$ & -..-- & $-\cdots--$ \\
\hline
\end{tabular}

Fuente: Elaboración propia.

Durante los años en análisis, se evidencia claramente una continuidad que se quiebra con el cambio de gestión. Por un lado y desde el inicio de 2008, se advierte el aumento de los

\footnotetext{
${ }^{16}$ No hay datos ya que no se ejecutó la política.

${ }^{17}$ Datos obtenidos de la página http://datos.laplata.gov.ar/. Acceso en: 18 ago. 2017. Hoy el link es https://www.datos.laplata.gov.ar/ Acceso en: 18 nov. 2018.

${ }_{18}$ Hasta el momento de finalización de la presente investigación, no se registraron acciones ni información al respecto.

${ }^{19}$ En millones de pesos.

${ }^{20}$ Este número proviene de las entrevistas realizadas a diversos funcionarios actuales, aún cuando no hay dato publicado que lo respalde.

${ }^{21}$ Participación media en términos de votación en la Consulta Popular.
} 

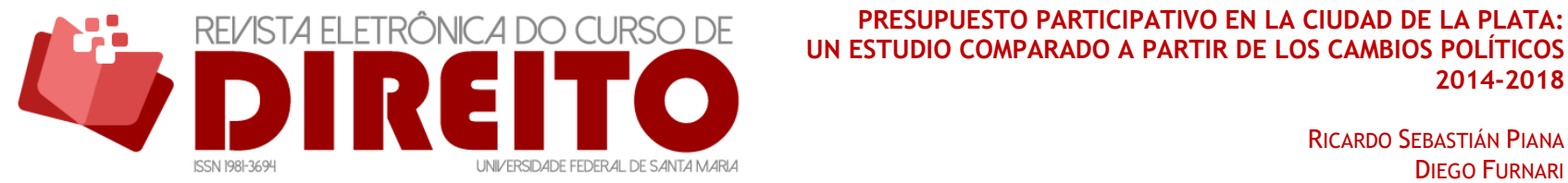

fondos públicos destinados a esta política (sobre todo en 2015) pero hay una fuerte caída en la cantidad de participantes tanto en las asambleas (instancias de participación directa) como de la votación. A partir de 2017, por primera vez desde 2008, se redujo el presupuesto destinado a ésta política pública a 98 millones de pesos (150 millones fueron en 2015 ) de los cuales el $50 \%$ se divide en partes iguales para cada sección (25), mientras que el otro $50 \%$ se destinará de acuerdo a las necesidades de cada zona y a la densidad poblacional. Pero el dato central es la ausencia oficial de información respecto a la participación, aún cuando se utilizaron las plataformas electrónicas.

\section{CONCLUSIÓN}

Algunas reflexiones del caso, a partir de la observación de ciertos resultados y aspectos, pueden dividirse de acuerdo a dos dimensiones centrales: por un lado la dimensión comunicacional/discursiva a partir de la cual es posible identificar las intenciones y los discursos que giraron en torno a las políticas participativas en las gestiones en análisis; por otro lado, la dimensión operativa, es decir, las medidas concretas que se fueron tomando y su impacto en la realidad de las políticas participativas en la ciudad. La puesta en común de ambas variables nos permitirá obtener las conclusiones finales del presente paper.

a)

2014-2015

a. En cuanto a los aspectos discursivos durante este período:

i. Se pudo observar el lugar secundario que la gestión encabezada por el ex Intendente Pablo Bruera le otorgó a las políticas o iniciativas participativas en cuanto a sus estrategias comunicacionales. Pocas o nulas referencias en las aperturas de cesiones del Poder Legislativo local (sobre todo los últimos dos años en donde las prioridades estuvieron basadas en el aspecto de seguridad y en las críticas recibidas tras las inundaciones del 2 de abril); escasa presencia de funcionarios de segundo o tercer rango en los medios de comunicación;

ii. Evidentemente, y como consecuencia de una política pública consolidada, la "gestión Bruera" no consideraba prioritario incorporar nuevas perspectivas o propuestas participativas en sus estrategias de campaña (especialmente, en la de 2015).

iii. La gestión no generó una estrategia comunicacional de difusión del Presupuesto Participativo que permitiese la incorporación de nuevos actores o multiplicase la 

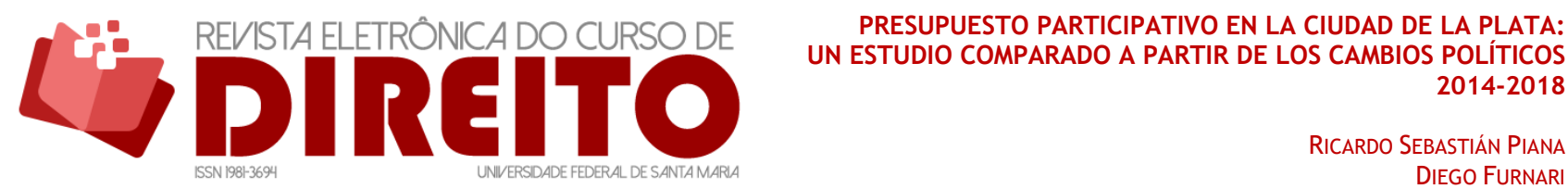

cantidad de vecinos interesados en participar. Incluso, la difusión de las fechas para las Asambleas, los resultados de las mismas e incluso la realización o no de las obras, pasaron casi desapercibidas a excepción de la intervención de algún medio de comunicación local. Incluso el sitio web presentaba características propias de una web 1.0 por su falta de dinamismo, un usuario pasivo, falta de interacción entre usuario y productor de contenidos, diseño poco amistoso.

b. En cuanto a los aspectos operativos durante este período:

i. Detectamos una tendencia a la baja en la participación: iniciada en 2008, la cantidad de proyectos presentados y asistencia a Consulta Popular, había crecido en forma constante, hasta el último bienio 2014-2015. Si bien se registró en 2015 el mayor número de asistentes a las asambleas barriales desde la implementación en 2008 con 6.000 vecinos y un aumento respecto de 2014 en la cantidad de votantes: de 32.198 a 34.000, la votación s/población, que había llegado a su pico en 2013 (10,4\%), disminuyó a casi la mitad en 2014 (6,5\%) y alcanzó al sólo el 8 \% de la población en 2015.

ii. Fuera del Decreto de 2008, durante la gestión de Bruera no existieron iniciativas ni políticas complementarias tendientes a ampliar los márgenes participativos ni a generar nuevas herramientas o espacios para que la ciudadanía pudiese informarse o generare nuevas propuestas. No se pensó ni se propuso, por ejemplo, en una manera ágil de abrir la información pública a los interesados ni espacios de control o auditoría que permitiesen una retroalimentación favorable para la toma de decisiones municipal. La poca información que se publicaba se limitaba a lo que la página web (sumamente precaria y poco interactiva) sugería respecto de los proyectos puestos a votación y posteriormente los resultados finales.

iii. El Presupuesto Participativo vio incrementado su partida presupuestaria desde su inicio hasta el final de este período.

b) $\quad 2016-2018$

a. En cuanto a los aspectos discursivos durante este período:

i. Discursivamente la nueva gestión convirtió los presupuestos del gobierno abierto en ejes fundamentales de su campaña electoral y como un instrumento inmediato de legitimación en sus primeros meses de gobierno. A su vez, la unificación de la estrategia comunicacional (traducida en productos comunicacionales) entre el nivel nacional, provincial y local, favoreció la aceptación social del discurso propuesto por el partido en el poder. 

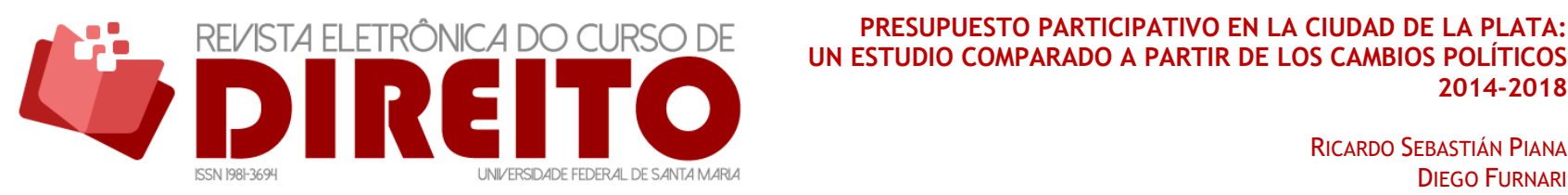

ii. No se logró explicar, suficientemente, la falta de aplicación de la experiencia en 2016. Ni el cambio de gestión, ni los supuestos errores del sistema anterior justifican la suspensión de la experiencia. Así mismo, pasado el primer semestre del 2018, el Municipio no había realizado ninguna acción comunicacional ni política vinculada a poner en marcha el Presupuesto Participativo del año 2018. Este reproche, le cabe también a la falta de pedidos de informes por parte de la oposición en el Concejo Deliberante así como de asociaciones civiles o ciudadanos: no hubieron reclamos concretos a este respecto, lo que tensiona la bidireccionalidad de esta política.

b. En cuanto a los aspectos operativos durante este período:

i. Se detectó una rápida reforma de las plataformas y canales de comunicación utilizados por el gobierno, quien transformó a las TICs (en especial las redes sociales como Twitter y Facebook) en la herramienta principal de comunicación. Desde allí generó un flujo informativo y de interacción con el vecino mucho mayor al que la ciudad venía acostumbrada.

ii. Se modernizó la vieja web y la reemplazó por una plataforma que permitiría una relación bidireccional con los vecinos y un mayor acceso.

iii. La falta de datos de la experiencia de 2017 deslegitima todas las bondades del nuevo sistema. En efecto, más allá de los aspectos positivos que presenta la plataforma https://presupuestoparticipativo.laplata.gob.ar, no hay información referida a la cantidad de votos de los proyectos ganadores (sólo porcentajes), a los plazos de implementación, a lo que se ejecutó durante el presente año, entre otros aspectos. Sólo este año, apareció la opición de presentar proyectos.

iv. No se propició modificar la ordenanza municipal que formalizase los cambios sustanciales que se desarrollaron a partir del año 2017.

En definitiva, buscamos comparar dos gestiones de gobiernos provenientes de espectros políticos e ideológicos opuestos a partir de la continuidad de una política, el presupuesto participativo.

En ese sentido, es fundamental destacar las enormes distancias que existieron entre las gestiones de gobierno analizadas respecto a lo que se decía y lo que se hizo. Como quedó evidenciado, la gestión de Pablo Bruera se caracterizó por la escasa estrategia comunicacional pero con importantes (y contundentes) avances en materia de presupuesto participativo aunque redujo la apertura del sistema político casi exclusivamente a dicha alternativa de política 
pública. Desde lo comunicacional la gestión demostró importantes falencias, cuya superación hubiesen permitido un mayor avance, sobre todo cualitativo, en la participación ciudadana en el gobierno. No obstante, desde 2008 hasta la salida del gobierno de Bruera en 2015, se registraron los mayores avances en materia de Presupuesto Participativo (tanto en cantidad de participantes como en presupuesto asignado) sin que fuese necesario convertir al mismo en un elemento discursivo preponderante para su gestión de gobierno o campaña electoral.

Con el cambio de actores políticos, la gestión encabezada por Julio Garro se basó (incluso en la campaña) en una propuesta moderna para modificar las lógicas estatales del gobierno municipal en un Gobierno Abierto. Trasparencia, apertura de datos y participación aparecieron tanto en discursos de campaña como en la apertura de sesiones ordinarias del poder legislativo local y declaraciones en los medios de comunicaciones locales. Pero lejos de profundizar una política pública ya consolidada, la actual gestión la puso en duda, primero al no ejecutarla en 2016 (sin dar explicación alguna al respecto) y luego sin otorgar un marco de legalidad a los cambios de fondo implementados para el período 2017. La eliminación de las asambleas, principal instrumento de vinculación e interacción real entre vecinos y organizaciones, no deja de asombrar en el marco de una propuesta cuyo objetivo principal era la de aumentar los márgenes de interacción entre el gobierno y el ciudadano, de dotar de más y mejores instrumentos al vecino para mejorar la calidad democrática y de gestión. Ello, junto a la ausencia de acciones durante el primer semestre de 2018, conllevan a intuir una serie de contradicciones muy importantes entre lo que la gestión prometió, sugirió o promovió (el dicho) y lo que realmente ejecutó, propuso o implementó (el hecho).

\section{REFERÊNCIAS}

ANNUNZIATA, Rocío. Los mecanismos participativos como puesta en escena de la proximidad: la experiencia del Municipio de Morón. In: NARDACCHIONE, Gabriel (comp.), Todos juntos. Dispositivos de participación de los gobiernos locales en la Argentina reciente. Buenos Aires, Argentina: Prometeo-UNGS, 2011.

ANNUNZIATA, Rocío. Decisión y deliberación en las formas no electorales de participación en Argentina: el caso del presupuesto participativo. Estudios Políticos, № 43, pp. 115-135, 2013.

BALARDINI, Sergio y Torrillate, Fernando. Municipio de Morón. 10 años de una exitosa gestión progresista. Morón. Buenos Aires, Argentina: Fundación Friedrich Ebert, 2009. Disponible en <http://library.fes.de/pdf-files/bueros/argentinien/06897.pdf> Acceso en: 10 oct. 2018. 
BLOJ, Cristina. Itinerarios de deliberación ciudadana. El programa Presupuesto Participativo del Municipio de Rosario Argentina. Revista Iberoamericana. América Latina - 356 España-Portugal, $N^{\circ} 32$, pp. 31-50, 2008

BRUERA, Pablo. Dijimos que veníamos a cambiar la forma de administrar el Municipio a partir de la idea de una comuna de puertas abiertas, sin exclusiones y este Presupuesto Participativo es un paso importante que estamos dando hacia ese objetivo. Diario El Día. La Plata, 22 febr. 2008. Disponible en: <https://www.eldia.com/nota/2008-2-22-con-criticas-bruera-lanzo-elpresupuesto-participativo>. Acceso en: 9 jun. 2018.

CALANNA, Emilio. Entrevista concedida a FURNARI, Diego. 14 de febrero de 2017.

CARMONA, Rodrigo. Descentralización y presupuesto participativo en ciudades metropolitanas: alcances y desafíos en un escenario de transformaciones Estado-Sociedad. In: Nardacchione, Gabriel comp, Todos juntos. Dispositivos de participación de los gobiernos locales en la Argentina reciente. Buenos Aires, Argentina: Prometeo-UNGS, 2011.

CARMONA, Rodrigo; MARTÍNEZ, Carlos. Luces y sombras del presupuesto participativo en la región metropolitana de Buenos Aires. Revista Región y sociedad, Volumen $26 \mathrm{~N}^{\circ}$ 61, pp. 267308, 2014.

\section{CONSEJO LATINOAMERICANO DE ADMINISTRACIÓN PARA EL DESARROLLO - CLAD. Carta Iberoamericana de Gobierno Abierto. 2016. Disponible en < https: / /www.clad.org/images/declaraciones/CIGA-Octubre-2016.pdf>. Acceso en: 10 sep. 2018.}

FURNARI, Diego. Un análisis Comunicacional sobre el presupuesto participativo en el barrio Altos de Hernández: Construyendo procesos de integración. Tesis de la Maestría en Planificación y Gestión de Procesos Comunicacionales. Universidad Nacional de La Plata, Buenos Aires, Argentina, 2014. Disponible en: <http://sedici.unlp.edu.ar/handle/10915/45550>. Acceso en: 19 ago. 2018.

GARRO, Julio. Con críticas a la gestión anterior, Garro presentó la nueva etapa del Presupuesto Participativo. Realpolitik. La Plata, 18 abr. 2017. Disponible en: < https://realpolitik.com.ar/nota/24045/con_criticas_a_la_gestion_anterior_garro_presento_la_n ueva_etapa_del_presupuesto_participativo/> Acceso en: 12 may. 2017.

GARRO, Julio. Los vecinos de La Plata ya pueden votar online las obras para su barrio. ADN Digital, La Plata, 20 abr. 2017. Disponível em: <http://andigital.com.ar/municipios/item/60371los-vecinos-de-la-plata-ya-pueden-votar-online-las-obras-para-su-barrio>. Acceso en: 9 jun. 2018.

LACARRIEU, Mónica. Construyendo participación y ciudadanía en el ámbito de las Comunas de ciudad de Buenos Aires. En Escolar, Marcelo, Badía, Gustavo y Federic Sabina Editores, Federalismos y descentralización en grandes ciudades: Buenos Aires en perspectiva comparada. Buenos Aires: Prometeo, 2004.

LA PLATA (ciudad). Datos abiertos. Disponible en: <https://www.datos.laplata.gov.ar>. Acceso en: 18 nov. 2018. 

UN ESTUDIO COMPARADO A PARTIR DE LOS CAMBIOS POLÍTICOS 2014-2018

RICARDO SEBASTIÁN PIANA DIEGO FURNARI

LA PLATA (ciudad). Presupuesto participativo. Disponible en: <https://presupuestoparticipativo.laplata.gob.ar>. Acceso en: 6 mar. 2018.

MATA, Ma. Cristina. Nociones para pensar la comunicación y la cultura masiva. Modulo 2. Curso de Especialización Educación para la comunicación. Buenos Aires: La Crujía, 1985.

OSZLAK, Oscar. Gobierno Abierto: Promesas, supuestos, desafíos. VIII Conferencia anual Inpae 2012: gobierno abierto: por una gestión pública más transparente, participativa y colaborativa. San Juan de Puerto Rico, Puerto Rico, 2012. Anales electrónicos. Disponible en: < http://www.oscaroszlak.org.ar/images/articulos-espanol/Gobierno\%20abierto.pdf> Acceso en: 9 sep. 2018.

OSZLAK, Oscar. Estado abierto: hacia un nuevo paradigma de gestión pública. En XVIII Congreso Internacional del Clad. Montevideo, Uruguay, 2013. Anales electrónicos. Acceso en: 9 sep. 2018.

OSZLAK Oscar y KAUFMAN Ester. Teoría y práctica del gobierno abierto: Lecciones de la experiencia internacional. GEALC-OEA-IDRC, 2014. Disponible en: <https: //redinpae.org/recursos/kaufman-oszlak.pdf>. Acceso en: 9 ago. 2018.

PAGANI, Ma. Laura. Vos proponés, vos decidís: Presupuestos participativos y participaciones ciudadanas en La Plata y Morón 2006-2014. Tesis de doctorado en Ciencias Sociales. Facultad de Humanidades y Ciencias de la Educación, UNLP, 2015. Disponible en: <http://hdl.handle.net/10915/50125> Acceso en: 15 ago. 2018.

PAGANI, Ma. Laura. Análisis de la implementación y resultados del presupuesto participativo en contextos locales: dos casos en Argentina. Cuadernos de Administración, Universidad del Valle, Dez 2016, Volumen 32 № 56, pp. 63-80, 2016.

PIANA, Ricardo Sebastián. Representación, selección, participación. In: CORBETTA, Juan Carlos, PIANA Ricardo Sebastián y MARCHIONNI José María Coords., Nuevos Ensayos sobre la democracia contemporánea. Buenos Aires, Argentina: JCC Editor. pp. 195-22, 2014.

PIANA Ricardo Sebastián y FURNARI, Diego. La participación como eje central del gobierno abierto. Las oportunidades para el municipio de La Plata. Revista Democracia Digital e Governo Eletrônico Volumen 2, º13, pp. 39-54, 2015.

PINILLOS, Cintia y SIGNORELLI, Gisela. Notas sobre participación y representación en el presupuesto participativo de la Ciudad de Rosario, Argentina 20022012. Postdata, Volumen $19 \mathrm{~N}^{\circ} 1$, pp. 45-70, 2014.

PLOT, Beatriz. Gestión pública y participación ciudadana en el municipio de La Plata. Geograficando, Vol. 1 № $^{\circ}, 2005$.

QUINTAR Aída. Presupuesto participativo en la Ciudad de Buenos Aires. A la búsqueda de una acción pública participativa. In: CATENAZZI, Andrea; QUINTAR, Aída; CRAVINO, María Cristina; DA REPRESENTAÇAO, Natalia; Novick, Alicia. El retorno de lo político a la cuestión urbana. Territorialidad y acción pública en el Área Metropolitana de Buenos Aires. Buenos Aires, Argentina: Ed. Prometeo- UGGS, 2009. 
RAMELLA, Sonia y GALVÁN Facundo. ¿Todos a votar? Participación ciudadana y sociedad civil en distintas experiencias de Presupuesto Participativo de gobiernos locales argentinos. Revista Miríada. Volumen 4, $\mathrm{N}^{\circ}$ 8. Ciudad Autónoma de Buenos Aires, Argentina: Universidad del Salvador, 2012.

RAMÍREZ-ALUJAS, Álvaro. Gobierno abierto y modernización de la gestión pública: tendencias actuales y el (inevitable) camino que viene. Reflexiones seminales. Revista Enfoques, Vol. IX, NN15, 2011, pp. 99-125. Disponible en < http://www.gigapp.org/administrator/components/com_jresearch/files/publications/Enfoques1 5_5_Ramirez-Alujas.pdf>. Acceso en: 18 nov. 2018.

ROMERO, Ricardo. Democracia participativa, una utopía en marca. Reflexiones, experiencia y un análisis del caso porteño. En Romero, Ricardo (comp.) Presupuesto Participativo Porteño en el contexto latinoamericano. Ciudad de Buenos Aires, Argentina: GEDEP. UBA, 2004.

SANTANA, Diego. Presupuesto Participativo en la Municipalidad de La Plata con utilización de nuevas tecnologías en la votación. En V Congreso de Administradores Gubernamentales. Administración Pública y Cuestión Federal: la red Nación, Provincias y Municipios. San Juan, Argentina, 2009. Anales electrónicos. Disponible en <https://goo.gl/iRbxd8 >. Acesso en:18 nov.2018

SCHILLAGI, Carolina. Devenir vecino-militante. Las asambleas barriales de Buenos Aires. En DELAMATA, Gabriela (comp.), Ciudadanía y territorio. Las relaciones políticas de las nuevas identidades sociales. Buenos Aires, Argentina: Editorial Espacio, 2005.

SORTINO, Carlos Entrevista concedida a FURNARI, Diego, 01 de junio de 2018.

SORTINO, Carlos. El Presupuesto Participativo como estrategia para la inclusión y la innovación políticas. La Plata, Argentina: Cooperativa Los Tilos, 2013.

URANGA, Washington 2005. Ciudadanía y espacio público: el derecho de los pobres a la información y a la comunicación. Disponible en <http://www.wuranga.com.ar/>. Acceso en: 10 sep. 2018.

\section{COMO FAZER A REFERÊNCIA DO ARTIGO (ABNT):}

PIANA, Ricardo Sebastián; FURNARI, Diego. Presupuesto participativo en la ciudad de la plata. Un estudio comparado a partir de los cambios políticos 2014-2018. Revista Eletrônica do Curso de Direito da UFSM, Santa Maria, RS, v. 13, n. 3, p. 1245-1267, dez. 2018. ISSN 1981-3694. Disponível em: <

https://periodicos.ufsm.br/revistadireito/article/view/34691 >. Acesso em: dia mês. ano. doi:

http://dx.doi.org/10.5902/1981369434691. 